“C 2019 IEEE. Personal use of this material is permitted. Permission from IEEE must be obtained for all other uses, in any current or future media, including reprinting/republishing this material for advertising or promotional purposes, creating new collective works, for resale or redistribution to servers or lists, or reuse of any copyrighted component of this work in other works." 


\title{
A Period-Reconfigurable Leaky-Wave Antenna with Fixed-Frequency and Wide-Angle Beam Scanning
}

\author{
Zheng Li, Member, IEEE, Y. Jay Guo, Fellow, IEEE, Shu-Lin Chen, Student Member, IEEE, Junhong \\ Wang, Senior Member, IEEE
}

\begin{abstract}
A novel fixed frequency beam scanning leaky-wave antenna based on a period-reconfigurable structure is presented. Operating at $5 \mathrm{GHz}$, the antenna consists of a slotted substrate integrated waveguide and 54 electrically small patches. Each patch element is etched with two dumbbell-shaped slots, and its operating state can be flexibly controlled by the biasing of PIN diode on a parasitic strip. An ideal array model employing isotropic point sources is used for the analysis on the scanning mechanism, based on which a new method for suppressing the higher-order space harmonics is developed. Using this method, the mono-harmonic radiation range can be dramatically extended, and a wide-angle beam scanning can be achieved by manipulating the period length of the leaky-wave antenna. An FPGA controlling platform is designed for the electronic control of the antenna. The measured results validate that the proposed antenna achieves good performance of wide-angle scanning $\left(125^{\circ}\right)$ with a peak gain of 11.8 $\mathrm{dBi}$ at a fixed frequency.
\end{abstract}

Index Terms-Beam scanning, leaky-wave antenna, periodic structure, reconfigurable antenna, space harmonic.

\section{INTRODUCTION}

L EAKY-WAVE antennas (LWAs) have attracted much interest in the electromagnetics community since the idea was proposed decades ago [1], [2]. It is a typical type of travelling-wave antenna with inherent features of frequency beam scanning, high gain, and low profile [3]. There have been extensive investigations on the LWAs with frequencydependent beam-scanning behavior from backward to forward, such as the periodic LWAs [4]-[10], and the composite right/left-handed (CRLH) LWAs [11]-[14]. However, most practical applications operate at specific frequency bands, such as radar detection, mobile communications and satellite communications. Therefore, the fixed-frequency beamscanning property would be preferred for antennas in these applications. To meet this demand, various principles and techniques have been proposed to realize fixed-frequency beam

This work was supported by the Australia Research Council under Grant DP160102219, and by the National Natural Science Foundation of China under Grant 61771038 and Grant 61871025. (Corresponding author: Zheng Li)

Z. Li and J. H. Wang are with the Key Laboratory of All Optical Network and Advanced Telecommunication Network, Ministry of Education, Institute of Lightwave Technology, Beijing Jiaotong University, Beijing 100044, China (e-mail: lizheng@bjtu.edu.cn;).

Y. J. Guo and S. L. Chen are with the Global Big Data Technologies Centre, University of Technology Sydney, Ultimo, NSW 2007, Australia (e-mail: Jay.Guo@uts.edu.au). scanning [15]-[21]. In [15], a half-width microstrip LWA loaded with lumped capacitors at the open edge is proposed, and the main beam can be steerable when the capacitors are electrically tuned to change the phase constant along the LWA. In [16] and [17], Fabry-Perot (FP) leaky waveguides loaded with tunable high impedance surface are designed, and the resonant condition of the leaky mode is changed using varactors on the high impedance surface, producing steerable beams. In [18], a LWA with fixed-frequency beam-scanning ability is proposed based on a half-mode substrate integrated waveguide (SIW). The dispersion behavior can be changed by loading series and shunt varactor diodes. In [19] and [20] electronicallyscanning LWAs is presented based on a CRLH transmission line loaded with varactors. Continuous scanning can be achieved by tuning the biasing voltage of varactors to change the dispersion behavior. In [21] a corrugated microstrip line loaded with varactor diodes is presented, in which periodic modulation of surface impedance is employed, and beam steering is realized by controlling the biasing voltage on the varactor diodes. The reported research in [18]-[21] is mainly focused on the manipulation of the phase constant of the fundamental mode of the LWAs.

In this paper, we present a method to achieve electronically controlled beam scanning by manipulating the period length of periodic LWAs, while the phase constant of the fundamental mode remains unchanged. Periodic LWAs are usually analyzed using the space harmonic method [3]. Multiple-harmonic radiation is often undesirable in a practical application, as multiple beams will be generated, and they cannot scan independently. Thus mono-harmonic radiation is usually favorable for practical periodic LWAs. However, the condition of mono-harmonic radiation can only be satisfied usually in a limited angular range. Therefore, in this paper, we present a new method of suppressing the higher-order harmonics for the purpose of extending the angular range of mono-harmonic radiation. Using this method, a wide scanning angle of $125^{\circ}$ is achieved in the measurement of the fabricated antenna.

The paper is organized as follows. In Section II the proposed antenna configuration and the mechanism of controlling the elements are described. In Section III the operation principle of the antenna is illustrated in detail using an ideal model, and a novel method for suppressing higher-order harmonics is developed. Then the activation states of the elements are summarized to produce intended steering beams. In Section IV 
the antenna is fabricated and measured, and an FPGA controlling platform is developed especially for electronically controlled beam steering. Both simulated and measured results have validated the proposed concept and antenna design. Conclusions are drawn in Section V.

\section{CONFIGURATION OF ANTENNA AND ELEMENT}

The configurations of the antenna and the element employed are described in detail in this section. Then the basic operation principle of the element is illustrated.

\section{A. Antenna Structure}

The antenna configuration is depicted in Fig.1(a). The substrates 1 and 2 are Rogers RT/duroid $6006\left(\varepsilon_{r}=6.15\right)$, with thickness of $2.54 \mathrm{~mm}$ and $1.27 \mathrm{~mm}$, respectively. The travelling-wave feeding structure is based on SIW, consisting of metal layers 1 and 2, and substrate 1, with waveguide width $w_{g}=24 \mathrm{~mm}$ as depicted in Fig.1(b). The thickness of the copper $(35 \mu \mathrm{m})$ is also considered in the model. Two rows of patch elements are located on metal layer 3 as radiating elements, and they are excited by the fields leaked out from the SIW through the coupling slots on metal layer 2. From Fig.1(b), it can be seen that there are 54 patch elements in total. The period for one row of elements is $10 \mathrm{~mm}$. By introducing an offset of $5 \mathrm{~mm}$ for the other row, an equivalent period $p_{0}=5 \mathrm{~mm}$ in $y$ direction is achieved, which is just half of the original period $10 \mathrm{~mm}$. This is very favorable for the period reconfiguration for the periodic LWA, because the smaller the $p_{0}$ is, the more lengths of the perturbation period $P$ for the LWA can be achieved, providing greater flexibility to achieve steering beams. This will be discussed in detail in Section III.

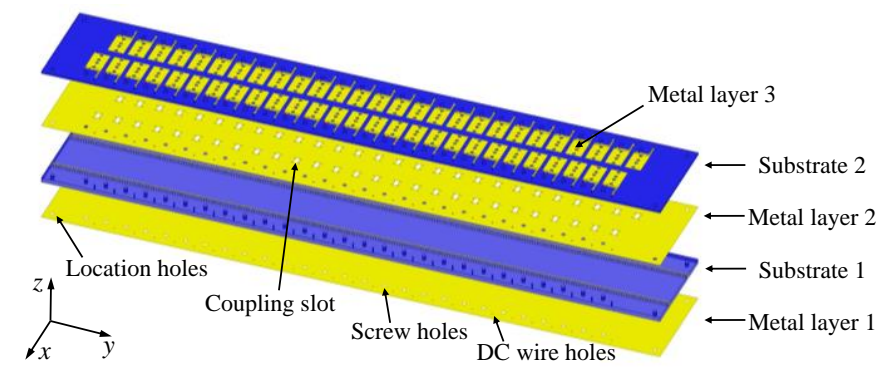

(a)

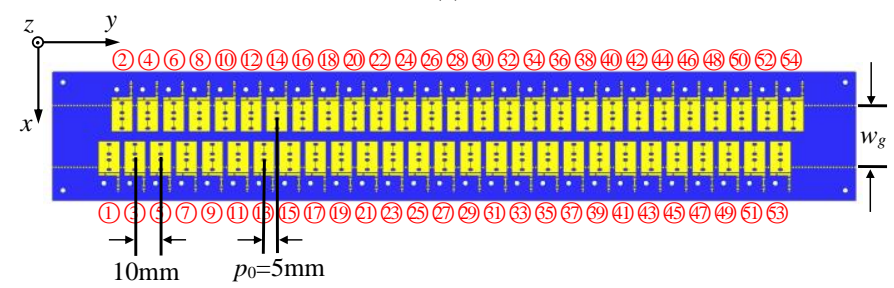

(b)

Fig. 1. Configuration of the LWA. (a) Overview (b) Top view

\section{B. Structure and Operation Principle of the Patch Element}

The structure of patch element is shown in Fig.2(a). There are two dumbbell-shaped slots symmetrically etched on the patch, and the patch is excited by an H-shaped coupling slot etched on the top of the SIW (metal layer 2). This patch has the advantage of smaller size compared with conventional patches.
In recent years, inspired by metamaterials, a number of electrically-small antennas have emerged [22]-[23]. In addition, it is an efficient way to realize miniaturization by etching slot with different shapes on the patch [24]-[26]. In this paper, we adopt these ideas in the patch design for reducing the patch size. An equivalent capacitance can be introduced by the narrow slot of the dumbbell, and an equivalent inductance can be produced by the metal portion beside the ellipses of the dumbbell-shaped slot. Therefore, an equivalent circuit model can be obtained as shown in Fig.2(b), generating a resonance at a specific frequency. A parasitic strip is located beside the patch. The shorted post at one end of the strip increases the equivalent resonant length of the strip to generate strong coupling with the patch, yielding a drift to the patch resonant frequency. The second role of the shorted post is to be connected in parallel as a common electrode for the biasing of the PIN diode (BAR5002L) on the parasitic strip, which works as a binary switch. When the diode is forward biased (using a resistance of $4 \Omega$ in the simulation), the switch in Fig.2(b) is on, and a strong coupling occurs between the patch and the parasitic strip, yielding a shift of patch operation frequency. On the other hand, if the diode is not biased (using a parallel connection of a capacitance of $0.08 \mathrm{pF}$ and a resistance of $3.5 \mathrm{k} \Omega$ ), the switch in Fig.2(b) is off, so the induced current on the parasitic strip is very weak, having little effect on the patch operating frequency. The DC biasing line is laid in $x$ direction, and is broken into sections with two inductors (0402HP-8N7X), in order to choke $\mathrm{RF}$ currents on it, and to reduce the cross-polarization.

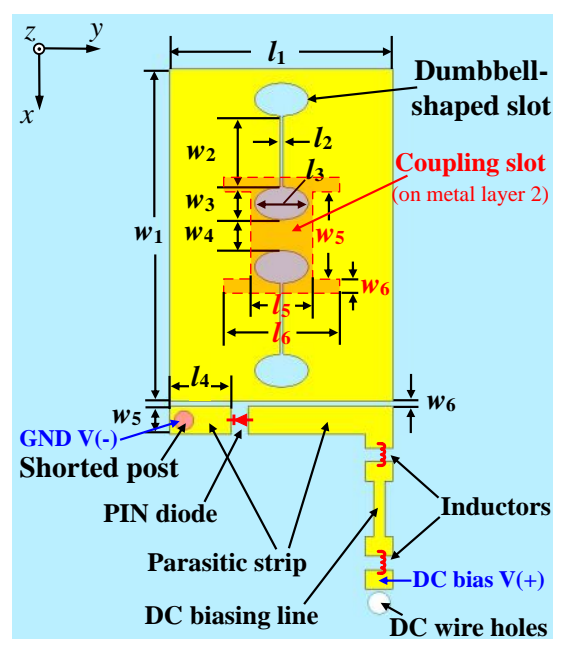

(a)

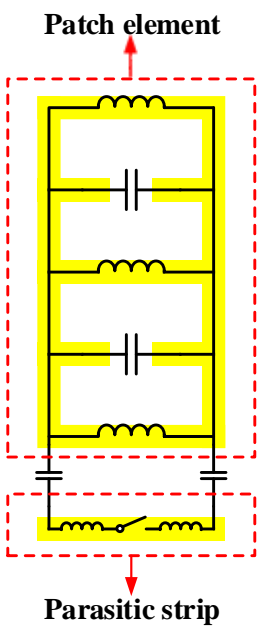

(b)
Fig. 2. Configuration and equivalent circuit of the element. (a) Element structure. (b) Equivalent circuit model. Structure parameters (mm): $w_{1}=12, w_{2}$ $=2.55, w_{3}=1.2, w_{4}=1.1, w_{5}=3, w_{6}=0.2, l_{1}=8, l_{2}=0.1, l_{3}=1.92, l_{4}=2.2, l_{5}=$ $2.1, l_{6}=4$.

The radiated power from one element is depicted in Fig.3, obtained with input power of $1 \mathrm{~mW}$. It can be seen that if the PIN diode is not biased (black curve), the operating frequency of the element is $5 \mathrm{GHz}$, where it shows a peak value of radiated power. If the PIN diode is forward biased (red curve), the operating frequency will drift to $5.2 \mathrm{GHz}$, while the radiated power at $5 \mathrm{GHz}$ is very low, so it can be regarded approximately 
as a non-radiative state at $5 \mathrm{GHz}$. Therefore, the binary element state can be switched between the activated state " 1 " (the diode is not biased) and the non-activated state " 0 " (the diode is forward biased), respectively. The advantage of this is that when the element is activated, the ohmic loss on the diode at 5 $\mathrm{GHz}$ (black curve in Fig.3) is very low due to small current on the diode. When the element is not activated (the diode is forward biased), the loss on the diode mainly happens at other frequency, yielding a lower peak value at $5.2 \mathrm{GHz}$ shown in the red curve. Therefore, the total loss at the operation frequency 5 $\mathrm{GHz}$ can be limited at a controllable level.

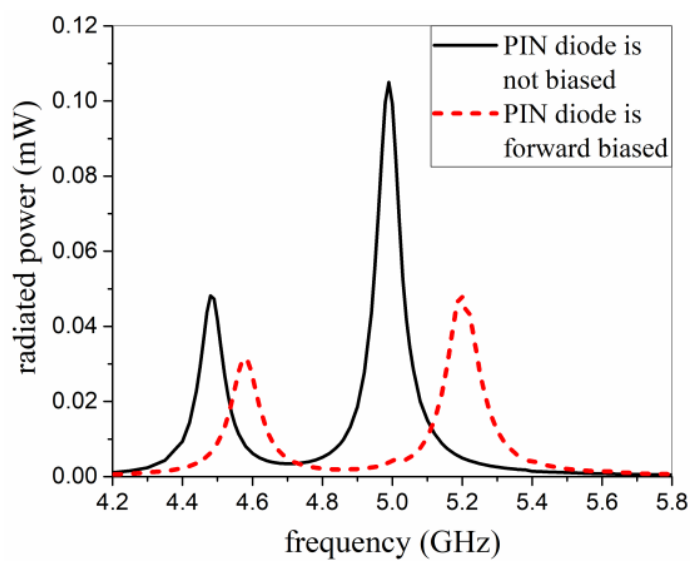

Fig. 3. Radiation efficiency of one unit cell when the diode is forward biased or not biased.

From Fig.3 it can be observed that there is another peak at $4.48 \mathrm{GHz}$ when the diode is not biased, and at $4.58 \mathrm{GHz}$ when the diode is forward biased, respectively. These peaks are caused by another resonant mode of the patch. Fig.4 shows the current on the patch at $5 \mathrm{GHz}$ and $4.48 \mathrm{GHz}$, respectively, when the diode is not biased. It can be seen that the mode in Fig.4(a) resonating in $y$ direction at $5 \mathrm{GHz}$ is the main mode of the patch, while the other mode in Fig.4(b) is generated by the resonance in $x$ direction (mainly determined by the length $w_{1}$ ).

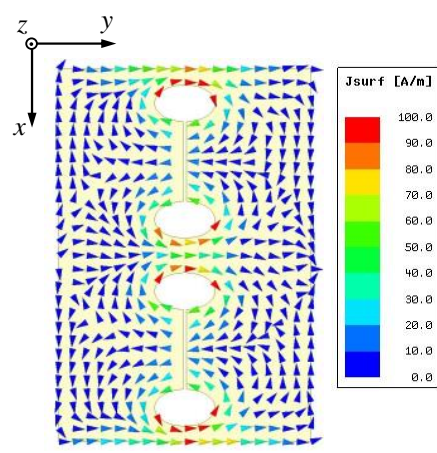

(a)

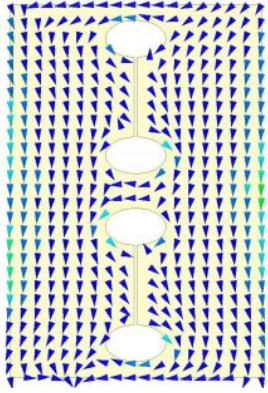

(b)
Fig. 4. Current distributions on the patch when the diode is not biased. (a) 5 $\mathrm{GHz}(\mathrm{b}) 4.48 \mathrm{GHz}$.

The proposed patch has both benefits and limitations. It has the advantages of more compact size, which is favorable to obtain more flexibilities for the period reconfiguration, and to achieve more beams to cover the full space. However, smaller bandwidth and higher loss may be caused as a result of the stronger resonance of the patch compared with the conventional patch. In addition, it may lead to more difficulties in the manufacture due to more complex structure of the element.

\section{PRINCIPLE ANALYSIS BASED ON IDEAL MODEL}

The radiation behavior of the periodic LWA presented in Fig. 1 can be analyzed approximately using a point-source array. The array element is assumed to be isotropic for easy analysis.

\section{A. Analysis on Mono-Harmonic Radiation}

Periodic LWAs always have periodic aperture fields. Using the space harmonic theory, the periodic aperture fields can be expanded into infinite terms of space harmonics [3], [27], in which the fast waves can radiate while the slow waves are bounded to the antenna aperture as surface waves. The phase constant for the $n^{\text {th }}$ harmonic can be expressed as

$$
\beta_{n}=\beta_{0}+\frac{2 n \pi}{P}
$$

where $\beta_{0}$ and $\beta_{n}$ are the phase constants of the basic harmonic mode and $n^{\text {th }}$ order harmonic mode. Then the scanning angle for each space harmonic can be approximately calculated using Eq.(2)

$$
\sin \theta_{n}=\frac{\beta_{n}}{k_{0}}
$$

where $\theta_{n}$ is the beam direction of the $n^{\text {th }}$ harmonic, and $k_{0}$ is the wavenumber in free space. Usually the $n=-1$ harmonic is selected to be the radiating mode, by choosing a slow-wave basic mode and a proper period length $P$. It is inferred from Eq. (1) that if $P$ can be manipulated, one would be able to control the beam direction $\theta_{n}$.

The transmission behavior of the SIW in Fig.1 is similar to that of the rectangular waveguide. An equivalent waveguide width of $23.6 \mathrm{~mm}$ can be achieved according to Eq.(9) in [28]. $\mathrm{TE}_{10}$ mode is the only transmission mode based on the dimension of the SIW, with the phase constant $\beta_{0}=223.2 \mathrm{rad} / \mathrm{m}$. By solving the following two equations

$$
\left\{\begin{array}{c}
-k_{0}<\beta_{-1}<k_{0} \\
\beta_{-2}<-k_{0}
\end{array},\right.
$$

we can get the mono-harmonic radiation condition as follows:

$$
\frac{2 \pi}{\beta_{0}+k_{0}}<P<\min \left\{\frac{2 \pi}{\beta_{0}-k_{0}}, \frac{4 \pi}{\beta_{0}+k_{0}}\right\}
$$

Substituting the values of $\beta_{0}$ and $k_{0}$ into Eq.(4), the monoharmonic condition is achieved as $19.2 \mathrm{~mm}<P<38.3 \mathrm{~mm}$. Fig. 5 shows the relationship between the scanning angle $\theta_{n}$ and the periodic length $P$, obtained using Eq.(1) and (2) (four harmonics $n=-1,-2,-3,-4$ are shown). It can be seen that the mono-harmonic radiation region (in grey color) is $P \in(19.2$ $\mathrm{mm}, 38.3 \mathrm{~mm}$ ), consistent with the result of Eq.(4), and the scanning range is from $-90^{\circ}$ to $34^{\circ}$. That is to say, if $P$ is greater than $38.3 \mathrm{~mm}\left(\theta_{-1}>34^{\circ}\right)$, the $n=-2$ harmonic will appear, and the mono-harmonic condition will be violated. In this case, therefore, the maximum scanning angle cannot break through the limitation of $34^{\circ}$.

Within the mono-harmonic radiation region, the normalized beam-steering radiation patterns are depicted in Fig.6. The period length $P$ must be an integer multiple of the element 
period $p_{0}$, so we select the values of $P$ to be $4 p_{0}(20 \mathrm{~mm}), 5 p_{0}$ $(25 \mathrm{~mm}), 6 p_{0}(30 \mathrm{~mm}), 7 p_{0}(35 \mathrm{~mm}), 8 p_{0}(40 \mathrm{~mm})$, and $9 p_{0}(45$ $\mathrm{mm})$, respectively. It can be seen that the beams (5) and (6) have obvious $n=-2$ harmonics in $-60^{\circ}$ and $-32^{\circ}$, respectively, since they are outside the mono-harmonic region. Therefore, using the $n=-1$ harmonic, only four beams (from (1) to (4)) can be generated before $n=-2$ harmonic occurs, with scanning angle range from $-60^{\circ} \sim 25^{\circ}$.

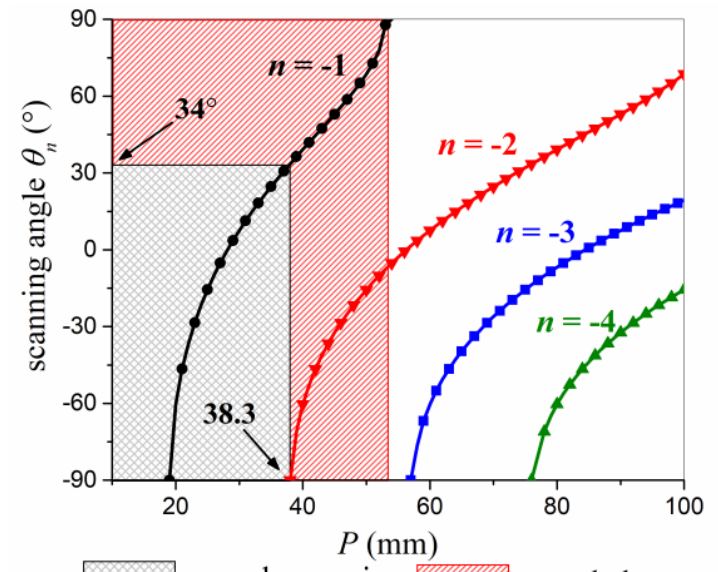

mono-harmonic scanning region

extended

scanning region

Fig. 5. Variation of the scanning angle $\theta_{n}$ and the periodic length $P$ for the four harmonics.

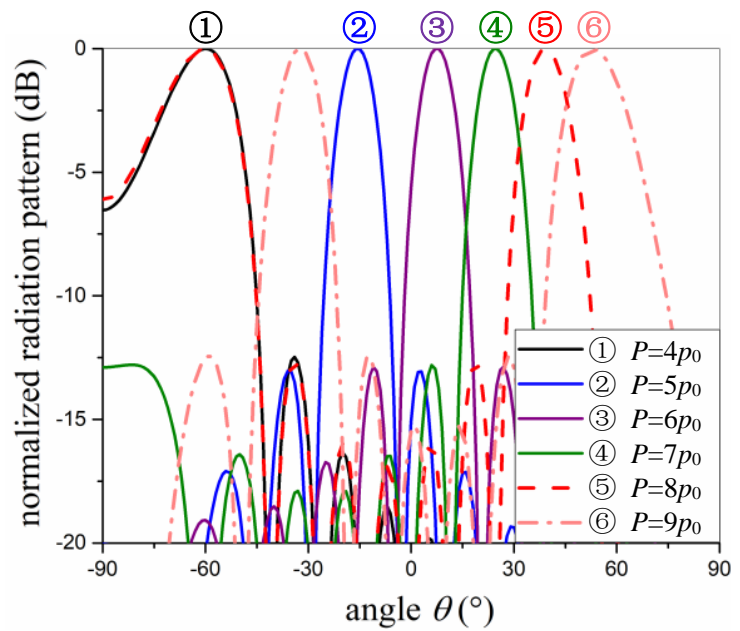

Fig. 6. Normalized radiation patterns of the point-source array when the period length $P$ is changed.

\section{B. Method for Suppressing Higher-Order Harmonics}

In order to overcome the limitation of mono-harmonic radiation, a method for suppressing the higher-order space harmonics is developed in this section. The aim is to extend the mono-harmonic scanning range to the red region in Fig.5. This method will be useful to increase the beam-scanning range for a periodic LWA.

The principle of the method is demonstrated in Fig.7. A new array of elements (in pink) is introduced in the structure, with a location shift $\Delta y$ from the original array (in red). Assuming that the $n^{\text {th }}$ harmonic is to be eliminated, we can obtain the phase relationship between the radiated fields of the original array and the newly added array in the beam direction of the $n^{\text {th }}$ harmonic as follows

$$
k_{0} \Delta y \sin \theta_{n}-\beta_{0} \Delta y= \pm(2 m+1) \pi, \quad m=0,1,2,3, \ldots
$$

where $(2 m+1) \pi$ means the phase difference is odd times of $\pi$. Substituting Eq.(1) and (2) into Eq.(5), we can get

$$
\Delta y= \pm \frac{(2 m+1) P}{2 n_{\text {sup }}}, \quad n_{\text {sup }}= \pm 1, \pm 2, \pm 3, \ldots
$$

where $n_{\text {sup }}$ is the order of harmonic that is to be suppressed. The values of $\Delta y$ for different harmonics are summarized in Table I. Therefore, by introducing a newly added array with a location shift $\Delta y= \pm \frac{(2 m+1) P}{2 n_{\text {sup }}}$ from the original array, the far-field radiation of the $n_{\text {sup }}$ harmonic can be eliminated.

TABLE I

LOCATION SHIFT OF THE NEWLY ADDED ARRAY

\begin{tabular}{|l|l|}
\hline \hline$n_{\text {sup }}$ & $\Delta y$ \\
\hline-1 & $\mp \frac{P}{2}$ \\
\hline-2 & $\mp \frac{P}{4}, \mp \frac{3 P}{4}$ \\
\hline-3 & $\mp \frac{P}{6}, \mp \frac{P}{2}, \mp \frac{5 P}{6}$ \\
\hline-4 & $\mp \frac{P}{8}, \mp \frac{3 P}{8}, \mp \frac{5 P}{8}, \mp \frac{7 P}{8}$, \\
\hline-5 & $\mp \frac{P}{10}, \mp \frac{3 P}{10}, \mp \frac{P}{2^{\prime}} \mp \frac{7 P}{10}, \mp \frac{9 P}{10}$ \\
\hline
\end{tabular}

For instance, if $n_{\text {sup }}=-1$, a new array of elements should be introduced with a location shift of $\Delta y=\mp \frac{P}{2}$. Similarly, the suppression of $n_{\text {sup }}=-2$ harmonic can be realized by choosing $\Delta y=\mp \frac{P}{4}$ or $\mp \frac{3 P}{4}$, which is very useful for a common periodic LWAs to extend the scanning range of mono-harmonic to the red region in Fig.5. In addition, if $n=-2$ and -3 harmonics exist simultaneously, the suppression of $n_{\text {sup }}=-3$ harmonic will help to add more mono-harmonic beams generated by $n=-2$ harmonic. It can also be observed that all the odd-order harmonics can be suppressed using $\Delta y=\mp \frac{P}{2}$. Theoretically, full-space continuous scanning (from $-90^{\circ}$ to $90^{\circ}$ ) can be achieved through this method, if the isotropic sources are employed, and arbitrary period $P$ is realizable. However, these two assumptions are difficult to achieve in practice. Therefore, the beam-scanning can be realized only in limited range, and the beams can only occur at discrete angles.

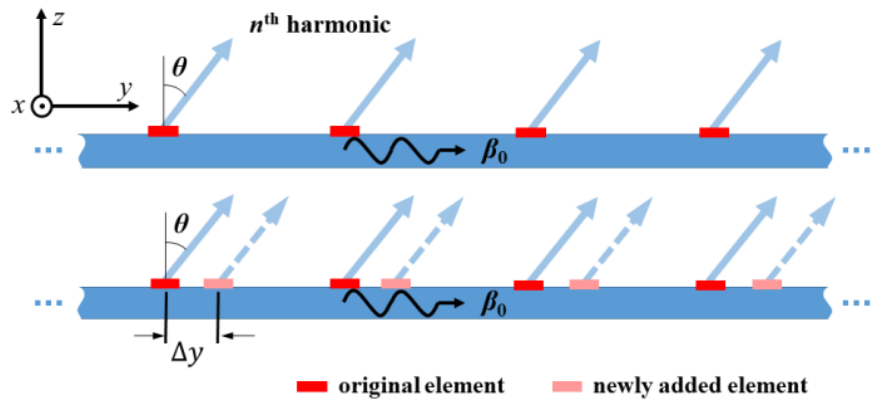

Fig. 7. Sketch of the method for suppressing the higher-order space harmonics.

The presented method can also be explained from the perspective of array factor (AF), as shown in Fig.8(a). Taking 
the case of $P=8 p_{0}$ as an example, we can choose seven radiating elements among the 54 elements of the LWA in Fig.1. This can be approximately equivalent to a seven-element array with element spacing $P=8 p_{0}$ and series-feeding phase constant $\beta_{0}=223.2 \mathrm{rad} / \mathrm{m}$, as shown in Fig.8(a) (marked in red). Then a new array (in pink) is introduced with a location shift $\Delta y$ from the original array. If these two arrays are regarded as two large "elements", a new $1 \times 2$ array can be achieved with a spacing $\Delta y=\frac{P}{4}$ between the two elements. Therefore, the radiation pattern of the total array can be seen as the product of the patterns of the large "element" and the AF.

Fig.8(b) shows the pattern of AF, while Fig.8(c) depicts the radiation patterns of the "element" and total array. In Fig.8(b) an obvious null in the direction $\theta=-60^{\circ}$ can be observed in the AF pattern, corresponding to the beam angle of $n=-2$ harmonic in Fig.8(c). Therefore, the product of the patterns of element and AF will result in a null in the total array pattern in $\theta=-60^{\circ}$, which is verified in the red curve in Fig.8(c). Meanwhile, in Fig.8(c) the beam generated from $n=-1$ harmonic in $\theta=39^{\circ}$ is not influenced because the $\mathrm{AF}$ is $0 \mathrm{~dB}$ in $\theta=39^{\circ}$ as shown in Fig.8(b). In short, the beam of $n=-2$ harmonic can be suppressed, and hence the $n=-1$ harmonic is the only radiating mode.

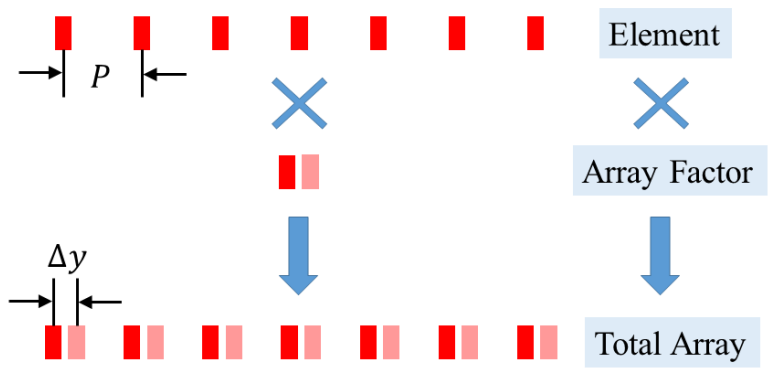

(a)

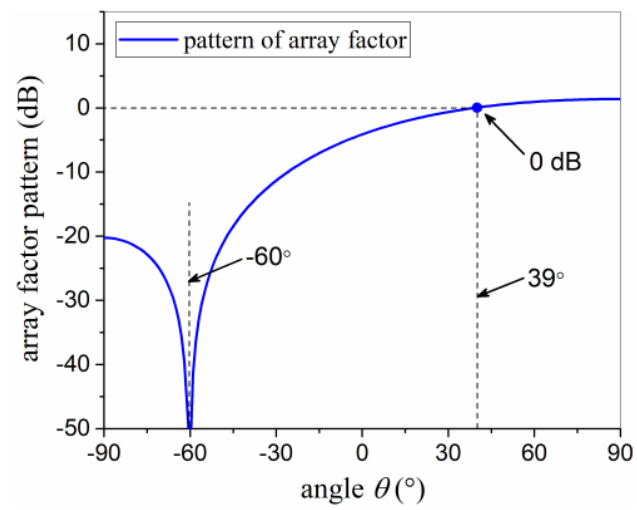

(b)

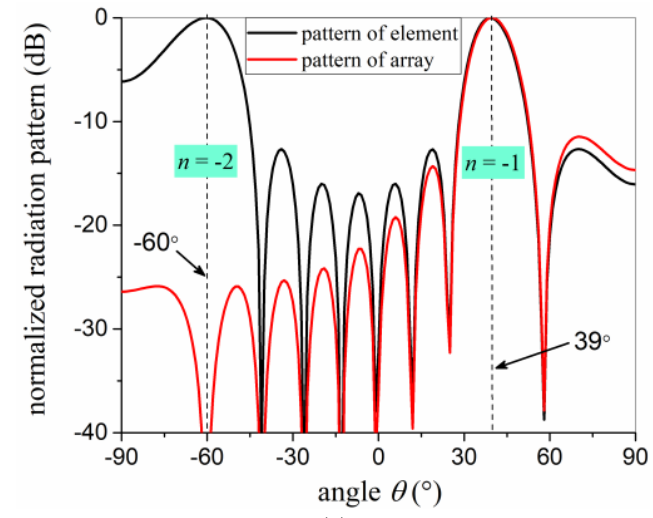

(c)

Fig. 8. Explanation on the presented method for suppressing $n=-2$ harmonic from the perspective of array factor $\left(P=8 p_{0}=40 \mathrm{~mm}\right)$. (a) Sketch of the principle (b) pattern of the array factor (c) patterns of the element and the total array.

Similarly, still taking the above case as an example, the method of suppressing $n=-1$ harmonic is verified using AF in Fig.9. By introducing a new array with a location shift $\Delta y=\frac{P}{2}$ from the original array, the newly constructed $1 \times 2$ array has an AF pattern as shown in Fig.9(a). It is obvious that a null is generated in the direction $\theta=39^{\circ}$, corresponding to the beam angle of $n=-1$ harmonic in Fig.9(b). Thus the suppression of $n$ $=-1$ harmonic is achieved in Fig.9(b). Meanwhile, in Fig.9(b) the beam of $n=-2$ harmonic in $\theta=60^{\circ}$ will be increased as a result of the AF in Fig.9(a). Therefore, the $n=-2$ harmonic becomes the only radiating mode, and mono-harmonic radiation is realized using this method.

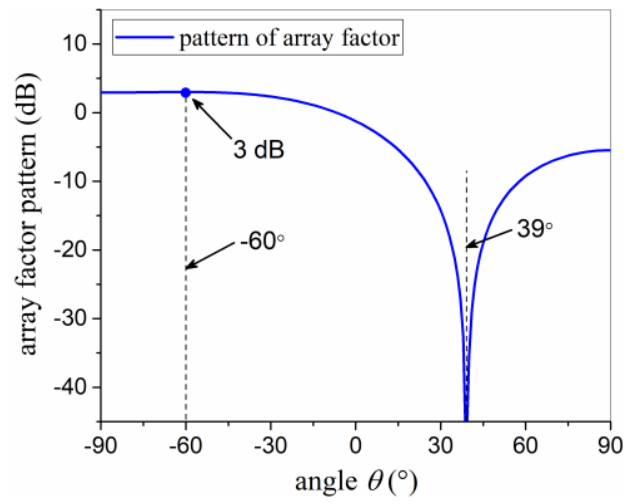

(a)

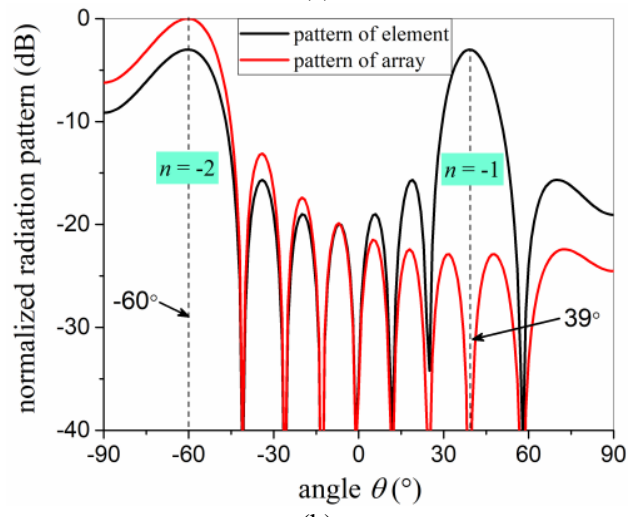

(b) 
Fig. 9. Explanation on the presented method for suppressing $n=-1$ harmonic $\left(P=8 p_{0}=40 \mathrm{~mm}\right)$. (a) pattern of the array factor (b) patterns of the element and the total array.

Therefore, it can be concluded that this method is efficient to overcome the limitation of mono-harmonic radiation, and hence the mono-harmonic beam-scanning region in Fig.5 can be extended to the red region.

\section{Element Activation States and Scanning Properties}

In order to suppress a certain harmonic, different activation states can be chosen. Here we give two examples to illustrate the principle of elements activation. Assuming that " 1 " and " 0 " indicate the element is activated and non-activated, respectively, and there are 54 elements in total.

\section{- Example 1: Suppressing $n=-2$ harmonic when $P=8 p_{0}$}

Assuming we have two states of element activation as follows, with the same period $P=8 p_{0}$.

State 1: $1010000010100000 \ldots$

State 2: $1111000011110000 \ldots$

In the original array, there is one unit activated in each period of eight units (marked as " 1 " in black) in State 1, while there are two units activated in State 2. For both states, the newly activated elements (marked as " 1 " in red) have a distance of $\Delta y=\frac{P}{4}=2 p_{0}$ from the original array. So both states can generate the same normalized radiation pattern with suppressed $n=-2$ harmonic (the red curve in Fig.8(c)). However, the radiation strengths are different due to different amounts of activated elements.

Using the method for suppressing the $n=-2$ harmonic, more beams can be achieved, extending the mono-harmonic radiation region into the red region in Fig.5.

- Example 2: Suppressing $n=-3$ harmonic when $P=15 p_{0}$

Although the scanning range has been extended using the method in Example 1, there is still more potential to achieve more beams to cover the full space, if mono-harmonic radiation can be realized by $n=-2$ harmonic. The case of $P=15 p_{0}$ is taken as an example to show the method for suppressing the $n=-3$ harmonic to realize mono-harmonic radiation of $n=-2$.

The original state for $P=15 p_{0}$ is as follows:

$100000000000000100000000000000 \ldots$

The normalized radiation pattern under this state is shown in Fig.10 (black curve). It can be seen that the pattern has three obvious beams at $\theta=32^{\circ},-16^{\circ},-90^{\circ}$, which are generated by $n$ $=-2,-3,-4$ harmonics, respectively. Although the $n=-4$ harmonic is still a non-radiative mode in Fig. 5 when $P=15 p_{0}=$ $75 \mathrm{~mm}$, it has already generated a strong backward lobe as a side lobe at $\theta=-90^{\circ}$.

In order to realize the mono-harmonic radiation of $n=-2$ harmonic, the $n=-3$ harmonic needs to be suppressed. It would be better if the lobe generated by $n=-4$ harmonic can be reduced at the same time. So the following four states are proposed for this purpose.

State 1: $101100000000000101100000000000 \ldots$

State 2: $111110000000000111110000000000 \ldots$
State 3: $111000001100000111000001100000 \ldots$ State 4: $111100001110000111100001110000 \ldots$

In States 1 and 2, we are using $\Delta y=\frac{P}{6}=2.5 p_{0}$ to suppress the $n=-3$ harmonic. The newly activated elements (marked as " 1 " in red) should have a location shift of $2.5 p_{0}$ from the original array (marked as " 1 " in black). However, " $2.5 p_{0}$ " is not an integer multiple of element spacing $p_{0}$, so in State 1 we have two new elements activated within one period of 15 elements, to produce an equivalent "center" with a distance of $2.5 p_{0}$ from the original activated element. Similarly, in State 2 there are three newly activated elements to generate an equivalent "center" with a distance of $2.5 p_{0}$ from the "center" of the first two elements. The normalized radiation patterns generated by States 1 and 2 are illustrated in Fig.10(a). It can be observed that the beam of $n=-3$ harmonic at $\theta=-16^{\circ}$ can be suppressed in both of these two states, but their radiation fields cannot be completely cancelled by each other in the beam direction of $n=$ -3 harmonic. The reason is that the number of newly activated elements is different from that of the original elements. It is noted that the reduction for the radiation of $n=-3$ harmonic is only $-8.2 \mathrm{~dB}$ in State 1, while it exhibits much better elimination in State 2. This is because that in State 2 the number of newly activated elements is relatively closer to that of the original elements (the number ratio is 3/2) than that in State 1 (the number ratio is 2/1), thus yielding a better result in State 2.

In States 3 and 4 , a location shift of $\Delta y=\frac{P}{2}=7.5 p_{0}$ is employed to suppress the $n=-3$ harmonic, so the newly activated elements have a location shift of $7.5 p_{0}$ from the original array. Similar to the above analysis, only the equivalent "centers" keep the spacing arrangement of $\Delta y$. The normalized radiation patterns generated by State 3 and 4 are illustrated in Fig.10(b). It can be observed that State 3 and State 2 (in Fig.10(a)) have the similar effects on the suppression of the beam in $\theta=-16^{\circ}$. Therefore, it is verified that both $\Delta y=\frac{P}{6}$ and $\Delta y=\frac{P}{2}$ can be used to suppress the radiation of $n=-3$ harmonic. It is worth noting that in State 4 not only the $n=-3$ harmonic but also the $n=-4$ harmonic can be dramatically suppressed. It is because that in State 4 the first four elements can be divided into two groups, and the "centers" of the first two and last two elements have a spacing of $2 p_{0}$, which is close to the requirement of suppression on the $n=-4$ harmonic $\left(\Delta y=\frac{P}{8}=\right.$ $\left.1.875 p_{0}\right)$. Similar things happen to the newly activated three elements (in red). Therefore, the radiation of $n=-4$ harmonic can be suppressed to a certain extent in State 4. However, due to the distinction between the practical $\Delta y$ and the desired $\Delta y$ $\left(1.875 p_{0}\right)$, the lobe of $n=-4$ harmonic cannot be completely eliminated. Finally we choose State 4 for the suppression of $n$ $=-3$ harmonic when $P=15 p_{0}$. 
TABLE II

ACTIVATION STATES OF ELEMENTS FOR DIFFERENT BEAMS

\begin{tabular}{|c|c|c|}
\hline Beam sequence number & Period length & Activation States \\
\hline (1) & $P=4 p_{0}$ & 110011001100110011001100110011001100110011001100110011 \\
\hline (2) & $P=4.25 p_{0}$ & 100010001100110001000100011001100010001000110011000100 \\
\hline (3) & $P=9 p_{0}\left(n_{\text {sup }}=-1\right)$ & 100011000100011000100011000100011000100011000100011000 \\
\hline (4) & $P=4.75 p_{0}$ & 100011000110001000010001100011000100001000110001100010 \\
\hline (5) & $P=5 p_{0}$ & 110001100011000110001100011000110001100011000110001100 \\
\hline (6) & $P=11 p_{0}\left(n_{\text {sup }}=-3\right)$ & 111000110001110001100011100011000111000110001110001100 \\
\hline (7) & $P=6 p_{0}$ & 110000110000110000110000110000110000110000110000110000 \\
\hline$(8)$ & $P=13 p_{0}\left(n_{\text {sup }}=-3\right)$ & 110000111000011000011100001100001110000110000111000011 \\
\hline (9) & $P=7 p_{0}$ & 111000011100001110000111000011100001110000111000011100 \\
\hline (10) & $P=15 p_{0}\left(n_{\text {sup }}=-3\right)$ & 001111000011100001111000011100001111000011100001111000 \\
\hline (11) & $P=8 p_{0}\left(n_{\text {sup }}=-2\right)$ & 011110000111100001111000011110000111100001111000011110 \\
\hline (12) & $P=9 p_{0}\left(n_{\text {sup }}=-2\right)$ & 001111000001111000001111000001111000001111000001111000 \\
\hline (13) & $P=9.5 p_{0}\left(n_{\text {sup }}=-2\right)$ & 111100000011100000011110000001110000001111000000111000 \\
\hline (14) & $P=10 p_{0}\left(n_{\text {sup }}=-2\right)$ & 110100000011010000001101000000110100000011010000001101 \\
\hline (15) & $P=10.5 p_{0}\left(n_{\text {sup }}=-2\right)$ & 011111000000111100000011111000000111100000011111000000 \\
\hline
\end{tabular}

Note: 1) "1" respresents that the element is activated (the diode is not biased). "0" respresents that the element is non-activated (the diode is forward biased).

2) The sequence of activation states coincides with the sequence number of elements in Fig.1(b).

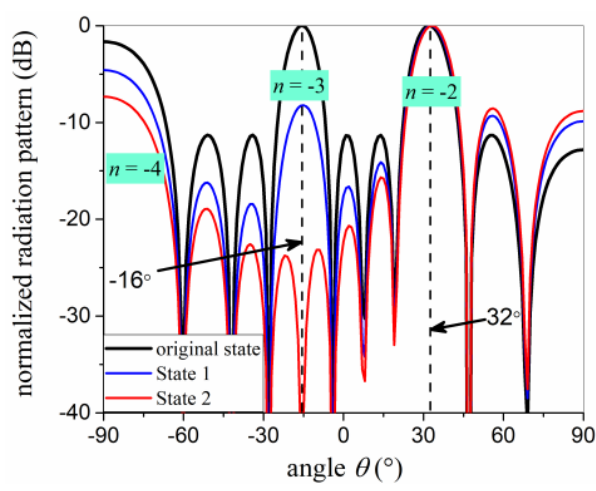

(a)

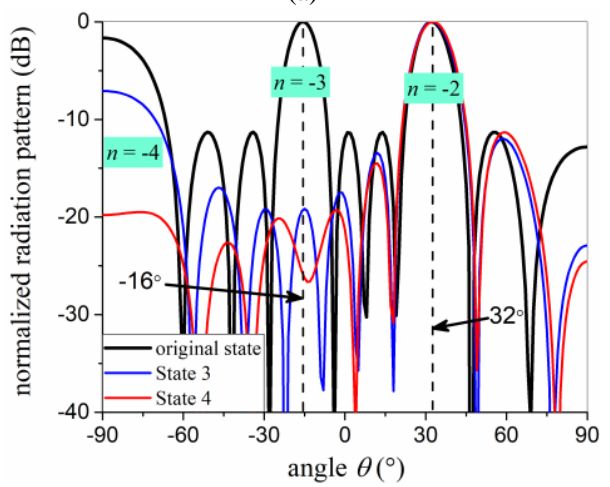

(b)

Fig. 10. Normalized radiation pattern for suppressing $n=-3$ harmonic $(P=$ $15 p_{0}$ ). (a) $\Delta y=\frac{P}{6}$ is employed in States 1 and 2. (b) $\Delta y=\frac{P}{2}$ is employed in States 3 and 4 .

Through the analysis of the above two examples, we can get the final activation scheme in Table II. For each period length $P$, by suppressing specific space harmonics, more beams can be achieved with mono-harmonic radiation. Totally 15 beams are selected to cover the scanning range, and each beam is marked with a sequence number to be easily distinguished. Fig.11 shows the normalized radiation patterns of mono-harmonic steering beams. The directions for the beams are illustrated in Fig.12, showing a scanning range from $-60^{\circ}$ to $78^{\circ}$, which is much wider than that in Fig.8. Note that all the above analysis is based on the assumption of isotropic point source with omnidirectional pattern. If the radiation pattern of element is in other types, the scanning range will be reduced to some extent.

In addition, it can be inferred that more beams can be generated if other non-integer period is chosen. On the other hand, if smaller element with smaller period $p_{0}$ can be generated, it would bring more flexibility to achieve more beams in desired directions.

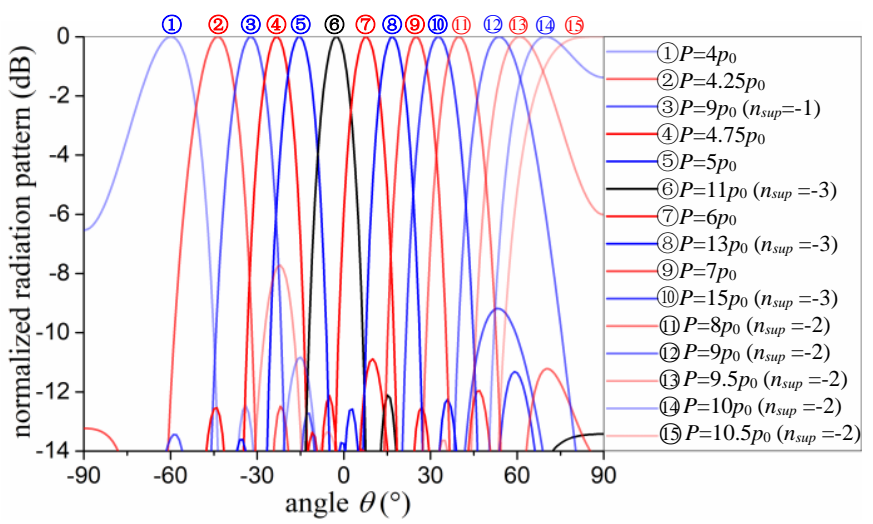

Fig. 11. Beam-scanning normalized radiation patterns of the isotropic-pointsource array when the harmonic suppression method is employed ( $n_{\text {sup }}$ is the order of the harmonic which is suppressed). 


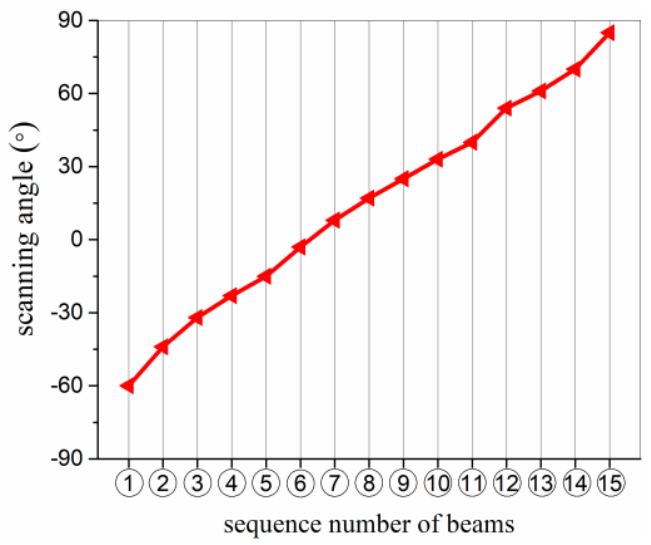

Fig. 12. Beam-scanning angle of each beam.

As a summary of this section, it can be concluded that the developed method for suppressing higher-order harmonics is of great significance to increase the beam-scanning range for periodic LWAs. This method has been verified by the ideal model of point-source array. In the next section this method will be employed in the practical periodic LWA.

By the way, it is worth mentioning that this method is also suitable for the suppression of grating lobes in antenna arrays, since all the analysis is based on an array model.

\section{RESUlTS AND DisCUSSION}

In this section the radiation performance of the proposed element is analyzed and the experimental results of a whole LWA are reported in detail. The conductor and dielectric losses have been considered in the simulation. An FPGA module is developed for controlling the operation state of the antenna. Experimental results are also given as a verification of the presented antenna.

\section{A. Radiation Pattern of Element}

In Fig. 2 due to the capacitance effect of the slot, the E field is mainly concentrated at the straight part with the length $w_{2}$ in the dumbbell-shaped slot. Therefore, the radiation of the patch is closer to the radiation from the magnetic currents at the slot. The radiation pattern of the presented element at $5 \mathrm{GHz}$ (when the diode is not biased) is shown in Fig.13. It is between the patterns of the isotropic point source (omni-directional pattern, black curve) and an infinitesimal electric current element source (blue curve). A very wide $3 \mathrm{~dB}$ beamwidth of $148^{\circ}$ can be observed in the pattern of the patch, which is important in achieving wide-angle beam scanning.

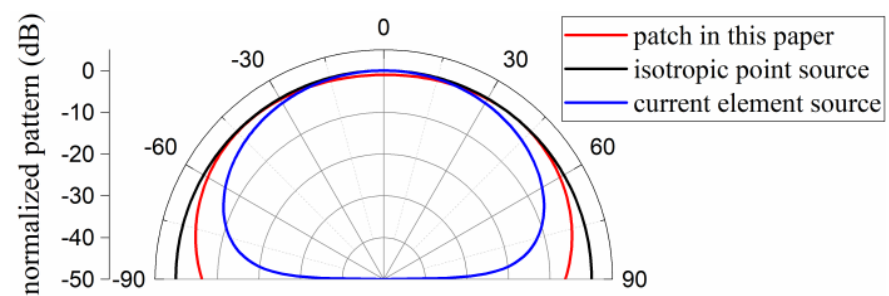

Fig. 13. Comparison of normalized radiation patterns of the presented patch, isotropic-point source, and current element source.

\section{B. Antenna Measurement with an FPGA Controlling Platform}

The beam-scanning LWA developed in this paper is fabricated through PCB processing, and the prototype is shown in Fig.14. The substrates 1 and 2 are shown in Fig.14(a), together with the FPGA controlling platform. Fig.14(b) shows the top and back views of the assembled antenna. The two substrates are mounted on an aluminum base for easy measurement. Two rows of nylon screws are aligned along the edges for stability reinforcement.

An FPGA platform is specially developed for controlling the antenna states. It is mounted on the bottom of an aluminum base for compact integration, as shown in Fig.14(b). An FPGA device (XC3S50AN-4TQG144C) is selected in the design. 54way voltage outputs are generated for controlling the 54 patch elements. Since all the diodes in the elements are connected in parallel in the biasing circuit, all the diodes share the same negative pole through the shorted post in each element in Fig.2(a), while the driving voltages are applied to the positive poles of diodes. 54 LED lamps are used for easily monitoring the activation states of diodes. Current-limiting resistors are employed to ensure the stability of LED lamps.

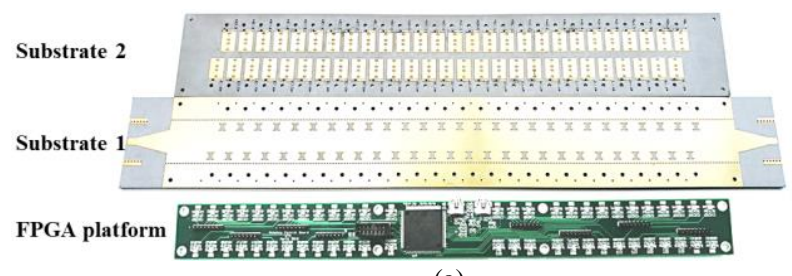

(a)

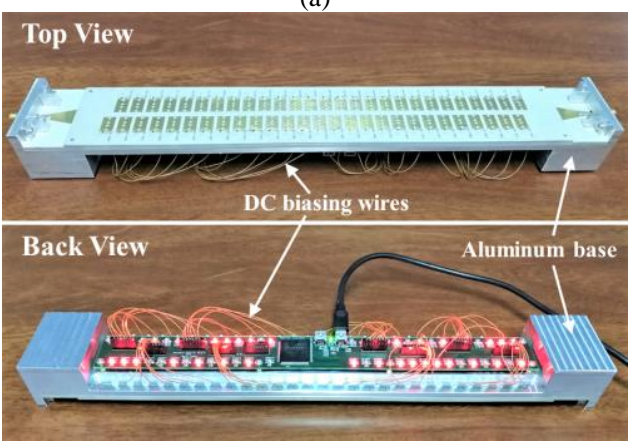

(b)

Fig. 14. Phototypes of the fabricated antenna. (a) Antenna substrates and FPGA platform (b) Overall view of the antenna.

\section{Radiation Patterns and S parameters}

The normalized beam-scanning radiation patterns are achieved using the activation states in Table II, and the simulated results are shown in Fig.15. In total, 15 beams are achieved in a scanning range of $137^{\circ}$ (from $-52^{\circ}$ to $85^{\circ}$ ). The broadside direction can be covered by the beam (6) (in black color). The measured radiation patterns are depicted in Fig. 16. We found that for all the activation states, the operating frequency corresponding to the measured peak gain shifted to 4.87 GHz. This can be attributed to the PCB processing inaccuracy. In addition, the chokes (inductors) operate as a parallel resonance of inductance and capacitance to generate very large impedance, while the adjacent parts on the board such as the solder joints have influences on the capacitance. 
Therefore, the chokes may have not provided the ideal isolation of RF currents, thus impacting the operating frequency. As a result, the measured radiation patterns in Fig.16 are given at $4.87 \mathrm{GHz}$. It can be observed that the measured scanning range is from $-60^{\circ}$ to $73^{\circ}$, showing a shift from the simulated scanning range.

It is worth mentioning that in this design only 15 beams are shown for clearly exhibiting the beam-scanning performance of the presented antenna. In fact, more beams can be produced to cover a given angular range by choosing proper lengths for the period $P$ of the LWA and using the developed method for suppressing undesirable harmonics in the meanwhile. This way, more steering beams at much denser angular intervals can be obtained.

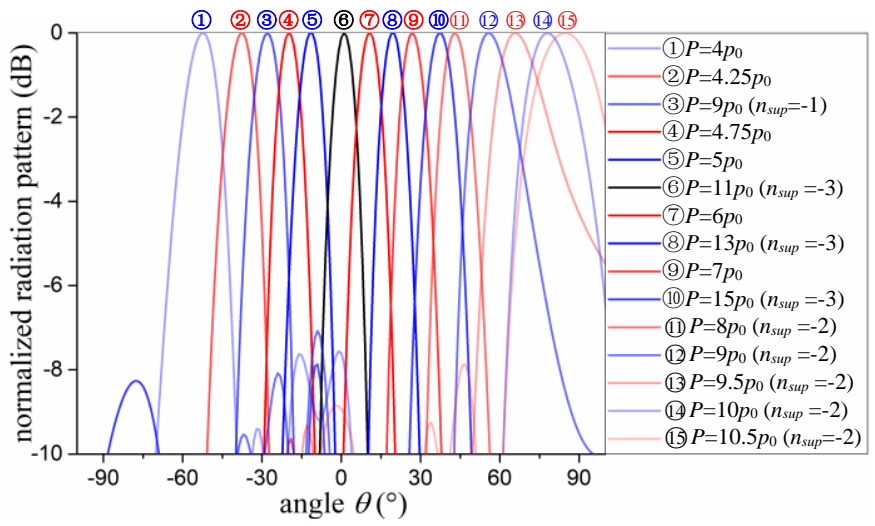

Fig. 15. Simulated results of the beam-scanning radiation patterns at $5 \mathrm{GHz}$ for the period reconfigurable LWA.

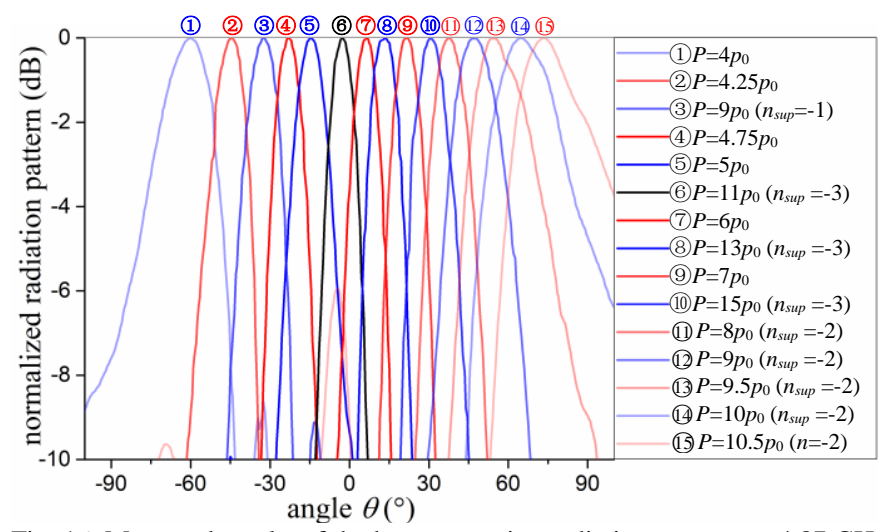

Fig. 16. Measured results of the beam-scanning radiation patterns at $4.87 \mathrm{GHz}$ for the period reconfigurable LWA.

The gain values in the main beam directions of all the patterns are shown in Fig.17. The simulated maximum gain is $12.5 \mathrm{dBi}$ corresponding to beam (7) in $\theta=11^{\circ}$. The gain for the broadside beam (6) is $12.0 \mathrm{dBi}$, with a small degradation caused by a slight effect of open-stop band. The degradation of gain for steering beams does not exceed $3 \mathrm{~dB}$ except the last beam (15). So the final $3 \mathrm{~dB}$ scanning range is $130^{\circ}$, from $-52^{\circ}$ to $78^{\circ}$, which is depicted directly in Fig.18. All the measured gains in Fig. 17 are obtained at $4.87 \mathrm{GHz}$ due to the aforementioned reason. The measured gain is lower than the simulated gain, indicating a degradation smaller than $1 \mathrm{~dB}$ in most angles. The measured gain for the last beam also shows a degradation exceeding $3 \mathrm{~dB}$, so the measured scanning range is $125^{\circ}$, from $-60^{\circ}$ to $65^{\circ}$, as shown in Fig.18. In Fig. 17 the beam directions of measured gain have a shift to the left compared with the simulated result because of the shifted operation frequency, which can also be observed in the beam-scanning angles in Fig.18.

In Figs.15 and 16 the side lobe levels (SLL) are not shown to avoid difficulty in distinguishing the patterns caused by the messy overlap of so many curves. So the SLLs of different beam-scanning patterns are supplemented in Fig.19. The SLLs for the beams (4) (9) (from $-20^{\circ}$ to $27^{\circ}$ ) are lower than $-10 \mathrm{~dB}$, while they are lower than $-7 \mathrm{~dB}$ for all the other beams.

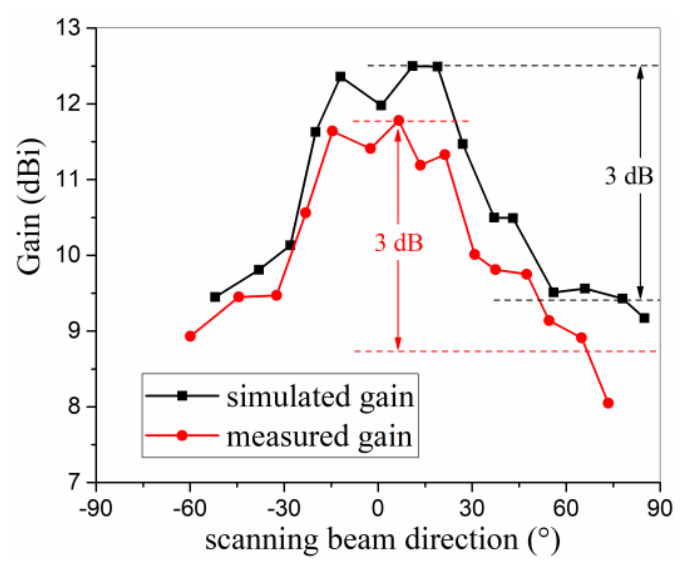

Fig. 17. The simulated gain at $5 \mathrm{GHz}$ and measured gain at $4.87 \mathrm{GHz}$ of each beam at the corresponding scanning angle.

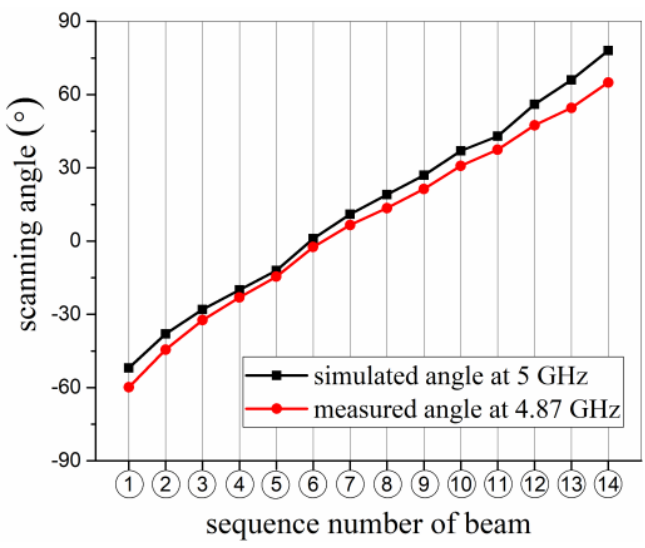

Fig. 18. The simulated and measured angles of the scanning beams.

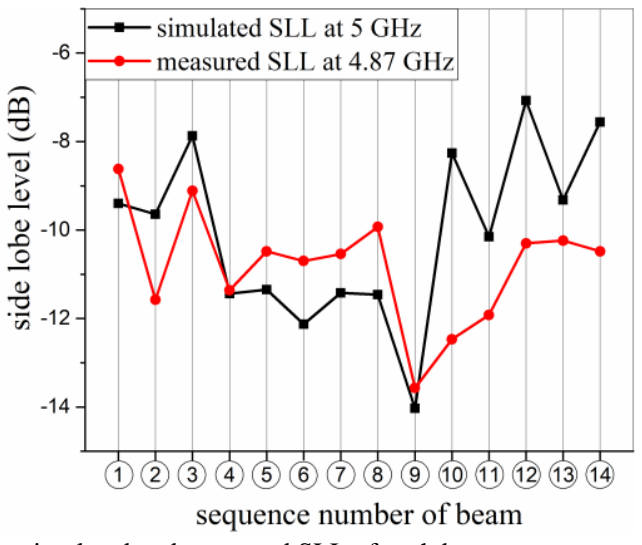

Fig. 19. The simulated and measured SLL of each beam. 
TABLE III

COMPARISON OF THE PRESENTEd AND REPORTED FIXED-FreQUENCY ELECTRICALLy SCANNING LWAs

\begin{tabular}{|c|c|c|c|c|c|c|c|c|}
\hline Ref. & Principle adopted & Structure type & $\begin{array}{l}\text { Antenna } \\
\text { length }\end{array}$ & Polarization & \multicolumn{2}{|c|}{ 3dB scanning range } & \multicolumn{2}{|c|}{ Peak Gain (dBi) } \\
\hline$[16]$ & Tuning on cavity resonant condition & FP cavity & $5 \lambda_{0}$ & linear & $33^{\circ}$ & $25^{\circ}$ & 12.6 & 12.95 \\
\hline$[17]$ & Tuning on cavity resonant condition & FP cavity & $5 \lambda_{0}$ & linear & $\begin{array}{c}120^{\circ}(\text { not } \\
3 \mathrm{~dB})\end{array}$ & $50^{\circ}$ & N/A & 11.6 \\
\hline$[18]$ & Manipulation on dispersion characteristic & $\begin{array}{l}\text { Half-Mode } \\
\text { SIW }\end{array}$ & $3.25 \lambda_{0}$ & circular & $92^{\circ}$ & $66^{\circ}$ & 11.3 & 9.89 \\
\hline$[20]$ & Manipulation on dispersion characteristic & CRLH & $2.8 \lambda_{0}$ & circular & $58^{\circ}$ & $57^{\circ}$ & 6.34 & 6.15 \\
\hline$[21]$ & Modulation on surface impedance & Microstrip line & $6 \sim 6.4 \lambda_{0}$ & linear & N/A & $45^{\circ}$ & N/A & 8 \\
\hline This work & Reconfiguration of period length & Slotted SIW & $4.6 \lambda_{0}$ & linear & $130^{\circ}$ & $125^{\circ}$ & 12.5 & 11.8 \\
\hline
\end{tabular}

It should be pointed out that, owing to the inherent dispersion characteristic of most leaky-wave structures, the beam direction of a leaky wave antenna will change over the operating band. For the antenna presented, the beam direction shifts are about $6^{\circ}$ in the boresight and this increases to about $9^{\circ}$ for beams with the widest scanning angles when the frequency changes from $4.9 \mathrm{GHz}$ to $5.1 \mathrm{GHz}$. This means that the antenna is more suited for operating within a narrower sub-band, which is not uncommon for modern communications systems. Further research is needed to make the antenna suitable for broadband systems.

Fig.20 shows the reflection coefficients of the LWA. In Fig.20(a) only five states are shown to demonstrate the behavior of the simulated reflection coefficients to avoid messy overlap of curves, and they are below $-10 \mathrm{~dB}$ at the operating frequency $5 \mathrm{GHz}$. The measured reflection coefficients are shown in Fig.20(b), and they are all under $-10 \mathrm{~dB}$ at the operation frequency $4.87 \mathrm{GHz}$. The open-stopband effect is not obvious from Fig.20. The reason may be that in the two-layer-substrate structure, substrate 1 has a relatively large thickness, yielding to smaller reflection at each slot compared with that in a conventional single-layer LWA. In addition, according to the impedance analysis in [29], the H-shaped coupling slot on the top of the SIW in this paper is consisting of both transverse and longitudinal slots, which may have better impedance matching performance than that by simply using only one transverse slot. However, from Fig.17 a degradation of gain at broadside can still be observed even though the requirement of $-10 \mathrm{~dB}$ has been satisfied in the reflection coefficients. Therefore, it can be inferred that by further optimizing the shape of the H-shaped coupling slot on the SIW, the impedance matching performance between the SIW, the slot and the patch element can be improved. In addition, there are some other method that may be adopted in this design, such as introducing a metallic post inside substrate 1 and beside the coupling slot as a tuning element (shunt inductance) to compensate the capacitance of the coupling slot for impedance matching [30]. By using these methods, it is expected that the open-stopband effect can be further suppressed, and the gain at broadside can be improved.
From Fig.20(c), it can be found that the magnitudes of $S_{11}$ for all the 15 states are below $-10 \mathrm{~dB}$ at the operating frequency for both the simulated and measured results. The magnitudes of $S_{21}$ for the simulated results exhibit a fluctuation between $-7 \mathrm{~dB}$ and $-10 \mathrm{~dB}$, while the measured values are slightly lower than the simulated ones.

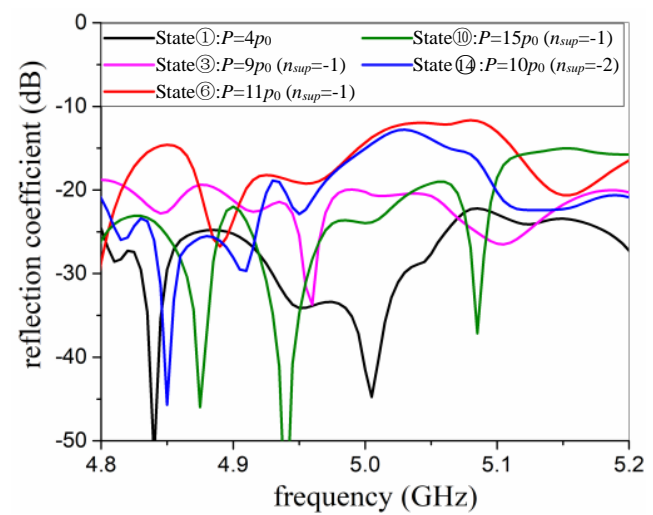

(a)

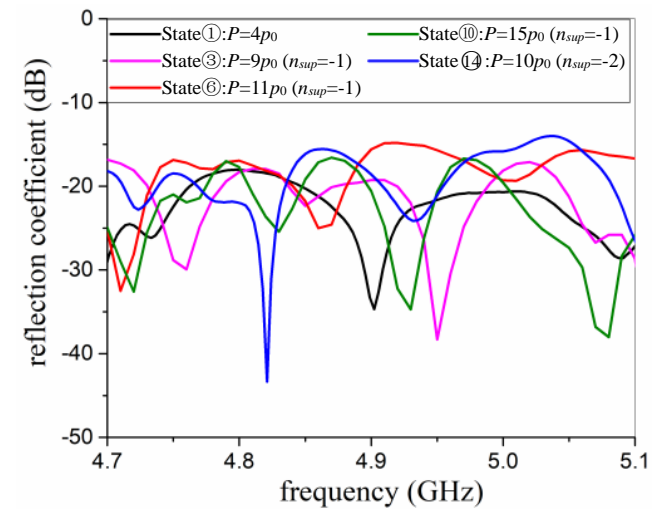

(b) 


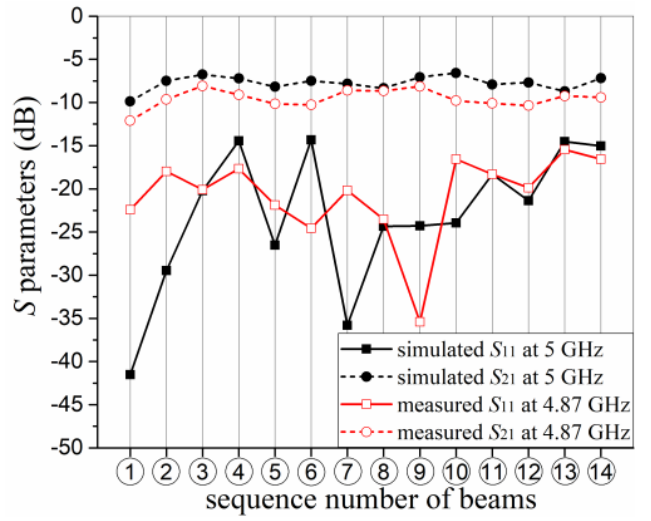

(c)

Fig. 20. $S$ parameters for the periodic LWA. (a) Simulated reflection coefficients. (b) Measured reflection coefficients. (c) $S$ parameters corresponding to each beam.

The attenuation constant $\alpha$ corresponding to each beam is calculated using the values of $S_{21}\left(\alpha \approx-\ln \left|S_{21}\right| / L\right)$, and the normalized result $\left(\alpha / k_{0}\right)$ is depicted in Fig.21. It can be observed that the simulated result of $\alpha / k_{0}$ fluctuates around 0.03 , which is relatively a small value, and is beneficial to achieve high directivity. The measured curve shows similar trend, but is a little higher than the simulated result.

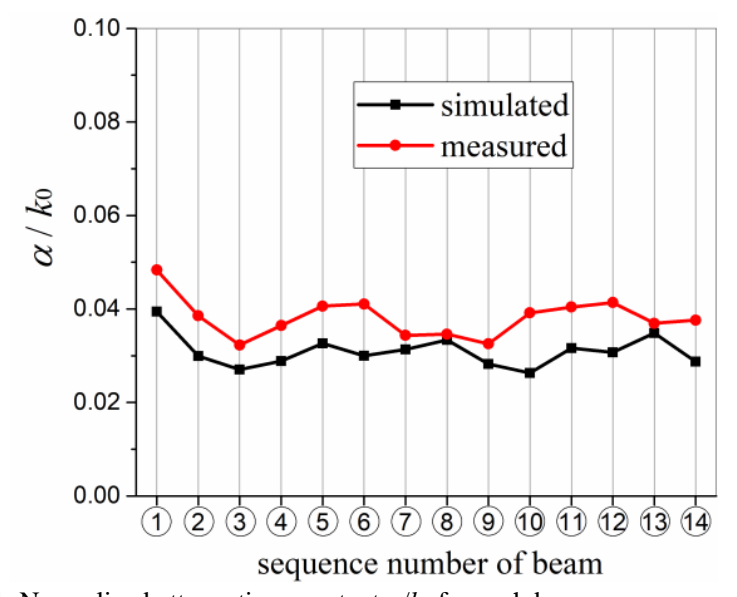

Fig. 21. Normalized attenuation constant $\alpha / k_{0}$ for each beam.

In order to study the antenna efficiency, the power loss of the proposed LWA is investigated using full-wave simulation, and the results are shown in Fig.22. The total stimulated power for the antenna can be divided into four parts: (i) the power reflected at the input port (which can be calculated from $S_{11}$ ); (ii) the power radiated into free space (which can be calculated from the integration of fields on the radiation boundary using HFSS full-wave simulation); (iii) the power absorbed at the terminal load (which can be calculated from $S_{21}$ ); (iv) the power lost on the structure (including diodes, conductor, and dielectric), which is calculated by subtracting (i) (ii) (iii) from the total stimulated power. Fig.22 shows the power percentages of part (iii) and part (iv). It can be observed that the power absorbed at the terminal load (part (iii)) is still a little high, varying from $16 \%$ to $21 \%$ for different beam directions. The power lost on the diodes, conductor and dielectric (part (iv)) is around $40 \%-46 \%$. The reason is that the resistance of the diodes generate high ohmic loss, and obvious losses will also be caused by the resonance on the copper patch and the loss tangent 0.0027 of the dielectric substrates. Therefore, usually it is quite difficult to reduce the power loss of part (iv) in a practical antenna. In addition, it is difficult to calculate the losses on diodes, conductor and dielectric separately. So here we give a total percentage of part (iv).

The antenna efficiency is also shown in Fig.22, varying from $36 \%$ to $43 \%$. There are some methods to increase the antenna efficiency: First, since there is still a certain amount of power remained at the end of the antenna (16\%-21\%), the $S_{21}$ can be made much lower and the antenna efficiency can be improved if we increase the antenna length and add more patch elements; Second, the antenna efficiency can be improved if low loss dielectric and better diodes can be used; Third, by modifying the shapes of the coupling slot and the patch, it may increase the percentage of radiated power, and hence improve the antenna efficiency.

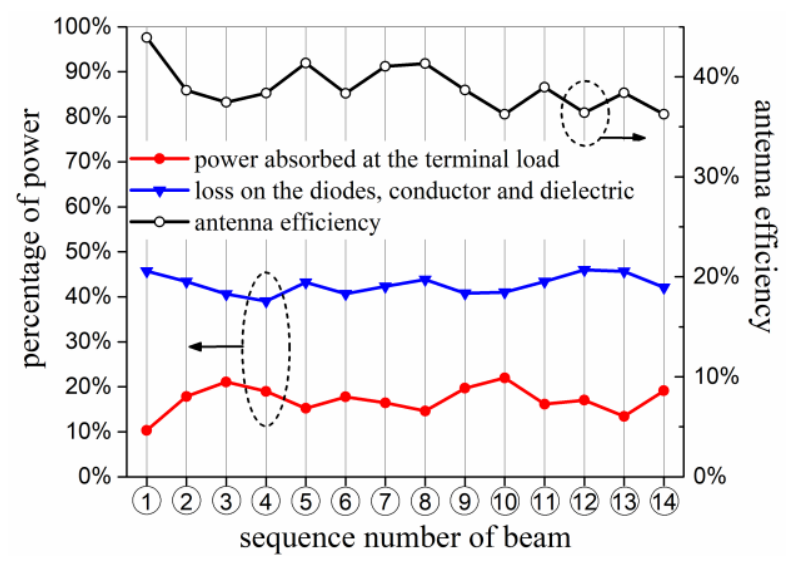

Fig. 22. Power percentage and antenna efficiency for each beam.

\section{Comparison with reported antennas}

A comparison of the reported fixed-frequency electrically scanning LWAs with the presented one is given in Table III. It is seen that the presented LWA in this paper has the advantages of wide scanning angle and consistent gain through the mechanism of reconfigurable period length.

\section{CONCLUSION}

In this paper an SIW-based period-reconfigurable LWA loaded with 54 patches is presented with electronically scanning characteristics, and the operating mechanisms are discussed in detail. The operating state of each patch element can be controlled by the biasing of the PIN diode. The beamscanning is realized by dynamic manipulation on the period length of the LWA. A new method for suppressing the higherorder harmonics is developed. Using this method, the monoharmonic scanning range can be significantly extended. The presented antenna is fabricated and measured. An FPGA platform is developed for electronically controlling beam of the antenna. The measured results show a wide scanning angle range of $125^{\circ}$. A $3 \mathrm{~dB}$ consistent gain is obtained in this 
scanning range, with a peak gain of $11.8 \mathrm{dBi}$. It is demonstrated that the experimental results have verified the design concepts, and the performances of the antenna. The developed antenna shows attractive features, and is expected to find applications in future wireless communications systems.

\section{ACKNOWLEDGEMENT}

The authors would like to thank Joseph Pathikulangara from CSIRO, Australia, for the development of the FPGA platform, and Hao Zhang from GBDTC, UTS, Australia, for the help of software debugging.

\section{REFERENCES}

[1] W. W. Hansen, Radiating Electromagnetic Waveguide (1940): U.S. Pat. $2,402,622$.

[2] L. O. Goldstone and A. A. Oliner, "Leaky-Wave Antennas-Part I: Rectangular Waveguides," IRE Trans. Antennas Propagat., vol. AP-7 (October 1959): 307-319.

[3] A. A. Oliner and D. R. Jackson, "Leaky-Wave Antennas," in J. L. Volakis (ed.), Antenna Engineering Handbook, Fourth Edition, New York, McGraw-Hill, 2007, Chapter 11.

[4] S. K. Podilchak, L. Matekovits, A. P. Freundorfer, Y. M. M. Antar, and M. Orefice, "Controlled leaky-wave radiation from a planar configuration of width-modulated microstrip lines," IEEE Trans. Antennas Propag., vol. 61, no. 10, pp. 4957-4972, Oct. 2013.

[5] Y. Li, M. F. Iskander, Z. J. Zhang, and Z. H. Feng, "A new low cost leaky wave coplanar waveguide continuous transverse stub antenna array using metamaterial-based phase shifters for beam steering," IEEE Trans. Antennas Propag., vol. 61, no. 7, pp. 3511-3518, Jul. 2013.

[6] J. H. Liu, Y. X. Li, and Y. L. Long, "Design of periodic shorting-vias for suppressing the fundamental mode in microstrip leaky-wave antennas," IEEE Trans. Antennas Propag., vol. 63, no. 10, pp. 4297-4304, Oct. 2015.

[7] G. Minatti, F. Caminita, E. Martini, S. Maci, "Flat optics for leaky-waves on modulated metasurfaces: adiabatic floquet-wave analysis," IEEE Trans. Antennas Propag., vol. 64, no. 9, pp. 3896-3906, Sep. 2016.

[8] D. K. Karmokar, Y. J. Guo, P. Y. Qin, K. P. Esselle, and T. S. Bird, "Forward and backward beam-scanning tri-band leaky-wave antenna," IEEE Antennas Wireless Propag. Lett., vol. 16, pp. 1891-1894, 2017.

[9] Y. L. Lyu, F. Y. Meng, G. H. Yang, D. Erni, Q. Wu, and K. Wu, "Periodic SIW leaky-wave antenna with large circularly polarized beam scanning range," IEEE Antennas Wireless Propag. Lett., vol. 16, pp. 2493-2496, 2017.

[10] Y. J. Li and J. H. Wang, "Dual-band leaky-wave antenna based on dualmode composite microstrip line for microwave and millimeter-wave applications," IEEE Trans. Antennas Propag., vol. 66, no. 4, pp. 16601668, Apr. 2018.

[11] L. Liu, C. Caloz, and T. Itoh, "Dominant mode leaky-wave antenna with backfire-to-endfire scanning capability," Electron. Lett., vol. 38, no. 23, pp. 1414-1416, Nov. 2002.

[12] S. Paulotto, P. Baccarelli, F. Frezza, and D. R. Jackson, "Full-wave modal dispersion analysis and broadside optimization for a class of microstrip CRLH leaky-wave antennas," IEEE Trans. Microw. Theory Tech., vol. 56 , no. 12, pp. 2826-2837, Dec. 2008.

[13] Nasimuddin, Z. N. Chen, and X. M. Qing, "Multilayered composite right/left-handed leaky-wave antenna with consistent gain," IEEE Trans. Antennas Propag., vol. 60, no. 11, pp. 5056-5062, Nov. 2012.

[14] A. Sarkar, M. Adhikary, A. Sharma, A. Biswas, M. J. Akhtar, Z. R. Hu, "Composite right/left-handed based compact and high gain leaky-wave antenna using complementary spiral resonator on HMSIW for Ku band applications," IET Microw. Antennas Propag., vol. 12, no. 8, pp. 13101315, Jun. 2018.

[15] R. O. Ouedraogo, E. J. Rothwell, and B. J. Greetis, "A reconfigurable microstrip leaky-wave antenna with a broadly steerable beam," IEEE Trans. Antennas Propag., vol. 59, no. 8, pp. 3080-3083, Aug. 2011.

[16] R. Guzmán-Quirós, J. L. Gómez-Tornero, A. R. Weily, and Y. J. Guo, "Electronically steerable 1-D Fabry-Perot leaky-wave antenna employing a tunable high impedance surface", IEEE Trans. Antennas Propag., vol.60, no.11, pp. 5046-5055, Nov. 2012.

[17] R. Guzmán-Quirós, J. L. Gómez-Tornero, A. R. Weily, and Y. J. Guo, "Electronic full-space scanning with 1-D Fabry-Pérot LWA using electromagnetic band-gap", IEEE Antennas Wireless Propag. Lett., vol. 11, pp. 1426-1429, 2012.

[18] A. Suntives and S. V. Hum, "A fixed-frequency beam-steerable halfmode substrate integrated waveguide leaky-wave antenna," IEEE Trans. Antennas Propag., vol. 60, no. 5, pp. 2540-2544, May 2012.

[19] S. Lim, C. Caloz, and T. Itoh, "Metamaterial-based electronically controlled transmission-line structure as a novel leaky-wave antenna with tunable radiation angle and beamwidth," IEEE Trans. Microw. Theory Tech., vol. 53, no. 1, pp. 161-173, Jan. 2005.

[20] J. H. Fu, A. Li, W. Chen, B. Lv, Z. J. Wang, P. Li, and Q. Wu, “An electrically controlled CRLH-inspired circularly polarized leaky-wave antenna," IEEE Antennas Wireless Propag. Lett., vol. 16, pp. 760-763, 2017.

[21] M. Wang, H. F. Ma, H. C. Zhang, W. X. Tang, X. R. Zhang, T. J. Cui, "Frequency-fixed beam-scanning leaky-wave antenna using electronically controllable corrugated microstrip line," IEEE Trans. Antennas Propag., 2018 (Early access).

[22] A. Erentok and R. W. Ziolkowski, "Metamaterial-inspired efficient electrically small antennas," IEEE Trans. Antennas Propag., vol. 56, no. 3, pp. 691-707, Mar. 2008.

[23] W. Lin and R. W. Ziolkowski, "Electrically small, low-profile, Huygens circularly polarized antenna," IEEE Trans. Antennas Propag., vol. 66, no. 2, pp. 636-643, Feb. 2018.

[24] R. H. Chen and Y. C. Lin, "Miniaturized design of microstrip-fed slot antennas loaded with C-shaped rings," IEEE Antennas Wireless Propag. Lett., vol. 10, pp. 203-206, 2011.

[25] M. K. Taher Al-Nuaimi and W. G. Whittow, "On the miniaturization of microstrip line-fed slot antenna using various slots," 2011 Loughborough Antennas \& Propagation Conference, Loughborough, UK, 2011.

[26] M. W. K. Lee, K. W. Leung, and Y. L. Chow, "Dual polarization slotted miniature wideband patch antenna," IEEE Trans. Antennas Propag., vol. 63, no. 1, pp. 353-357, Jan. 2015.

[27] Z. Li, J. H. Wang, J. J. Duan, Z. Zhang, and M. E. Chen, "Analysis on the radiation property of the bounded modes of periodic leaky-wave structure with finite-length using a hybrid method," Sci. Rep., 6:22917, 2016.

[28] F. Xu and K. Wu, "Guided-wave and leakage characteristics of substrate integrated waveguide," IEEE Trans. Microw. Theory Tech., vol. 53, no. 1, pp. 66-73, Jan. 2005

[29] Y. L. Lyu, X. X. Liu, P. Y. Wang, D. Erni, Q. Wu, C. Wang, N. Y. Kim, and F. Y. Meng, "Leaky-wave antennas based on noncutoff substrate integrated waveguide supporting beam scanning from backward to forward," IEEE Trans. Antennas Propag., vol. 64, no. 6, pp. 2155-2164, Jun. 2016.

[30] T. Mikulasek, J. Lacik, J. Puskely, and Z. Raida, "Design of aperturecoupled microstrip patch antenna array fed by SIW for $60 \mathrm{GHz}$ band," IET Microw. Antennas Propag., vol. 10, no. 3, pp. 288-292, Feb. 2016.

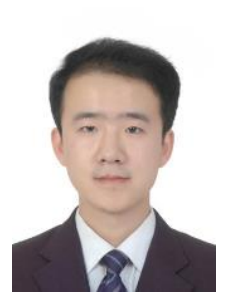

Zheng Li (M'15) received the B.S. degree in physics and $\mathrm{Ph} . \mathrm{D}$. degree in electrical engineering from Beijing Jiaotong University, Beijing, China, in 2006 and 2012, respectively. From 2008 to 2009, he was a visiting student with the Pennsylvania State University, Pennsylvania, USA. From 2017 to 2018, he was a visiting professor with the Global Big Data Technologies Centre, University of Technology Sydney, Australia.

In 2012, he joined the faculty of the Department of Electrical Engineering, Beijing Jiaotong University, where he became an associate professor in 2015. His research interests include electrically beamscanning antennas and leaky-wave antennas.

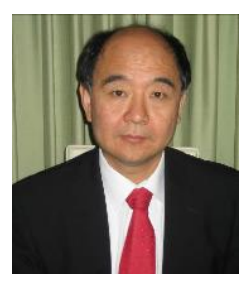

Y. Jay Guo (Fellow'2014) received a Bachelor Degree and a Master Degree from Xidian University in 1982 and 1984, respectively, and a PhD Degree from Xian Jiaotong University in 1987, all in China. His research interest includes antennas, mm-wave and $\mathrm{THz}$ communications and sensing systems as well as big data technologies. He has published over 400 research papers and holds 24 patents in antennas and wireless systems. $\mathrm{He}$ is a Fellow of the Australian Academy of 
Engineering and Technology, a Fellow of IEEE and a Fellow of IET, and a member of the College of Experts of Australian Research Council (ARC). He has won a number of most prestigious Australian national awards, and was named one of the most influential engineers in Australia in 2014 and 2015.

Prof Guo is a Distinguished Professor and the founding Director of Global Big Data Technologies Centre at the University of Technology Sydney (UTS), Australia. Prior to this appointment in 2014, he served as a Director in CSIRO for over nine years, directing a number of ICT research portfolios. Before joining CSIRO, he held various senior technology leadership positions in Fujitsu, Siemens and NEC in the U.K.

Prof Guo has chaired numerous international conferences. He is the Chair Elect of International Steering Committee, International Symposium on Antennas and Propagation (ISAP). He was the International Advisory Committee Chair of IEEE VTC2017, General Chair of ISAP2015, iWAT2014 and WPMC'2014, and TPC Chair of 2010 IEEE WCNC, and 2012 and 2007 IEEE ISCIT. He served as Guest Editor of special issues on "Antennas for Satellite Communications" and "Antennas and Propagation Aspects of 60$90 \mathrm{GHz}$ Wireless Communications," both in IEEE Transactions on Antennas and Propagation, Special Issue on "Communications Challenges and Dynamics for Unmanned Autonomous Vehicles," IEEE Journal on Selected Areas in Communications (JSAC), and Special Issue on " $5 G$ for Mission Critical Machine Communications", IEEE Network Magazine.

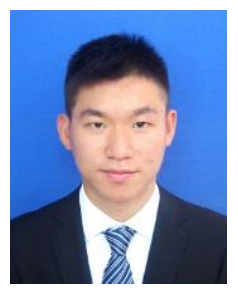

Shu-Lin Chen was born in Hubei Province, China. He received the B.S degree in electrical engineering from Fuzhou University, China, in 2012, and the M.S. degree in electromagnetic field and microwave technology from Xiamen University, China, in 2015. Since 2016, he has been working towards the Ph.D. degree in Engineering with the Global Big Data Technologies Centre (GBDTC), University of Technology Sydney (UTS), Australia.

His research interests include reconfigurable antennas, leaky-wave antennas, and adaptive array processing. He has authored or coauthored over 20 journal and conference papers. His paper was listed as Best Paper Award Finalists in the ISAP 2017, and as Honorary Mention Award in the APS-URSI 2017.

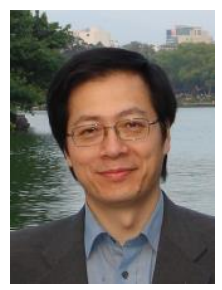

Junhong Wang (M'02-SM'03) was born in Jiangsu, China, in 1965. He received the B.S. and M.S. degrees in electrical engineering from the University of Electronic Science and Technology of China, Chengdu, China, in 1988 and 1991, respectively, and the Ph.D. in electrical engineering from Southwest Jiaotong University, Chengdu, China, in 1994. In 1995, he joined the faculty of the Department of Electrical Engineering, Beijing Jiaotong University, Beijing, China, where he became a Professor in 1999. From January 1999 to June 2000, he was a Research Associate with the Department of Electric Engineering, City University of Hong Kong, Hong Kong, China. From July 2002 to July 2003, he was a Research Scientist with Temasek Laboratories, National University of Singapore, Singapore. He is currently with the Key Laboratory of all Optical Network and Advanced Telecommunication Network, Ministry of Education of China, Beijing Jiaotong Univeristy, Beijing, China, and also with the Institute of Lightwave Technology, Beijing Jiaotong University, Beijing, China. His research interests include numerical methods, antennas, scattering, and leaky wave structures. 